\section{Physics in brief}

\section{N. David Mermin}

What Makes Nature Tick? By Roger G. Newton. Hanvard University Press: 1993. Pp 257. \$27.95, £22.25.

IN only a little more than 200 pages and with only an occasional equation, Roger Newton talks engagingly to his readers about Laplacian determinism, vectors, logarithms, differential calculus, time's arrow, Carnot cycles, thermodynamics, entropy, light, relativity, quantum theory, gauge fields and strings, waves, solitons, tachyons, elementary particles, bosons and fermions, phase transitions, superfluidity, superconductivity, symmetry, conservation laws, and many other things. If that sounds like a lot, that's because it is. Two questions come to mind: to whom is such a book addressed, and how well does it manage to convey a sense of the most important developments in physics from Isaac Newton to the frontiers of contemporary research?

According to its preface, the book grew out of the author's talks to a group of colleagues meeting to discuss their various disciplines, and it is intended for nonscientists who know very little mathematics. Roger Newton is thus addressing readers ignorant of technical physics, but at home with both abstract ideas and those aspects of contemporary physics that count as common knowledge among the educated. He is never patronizing, giving his readers a brisk, readable prose. They are clearly expected to catch on fast. You could not use this book as a text in an undergraduate course of the "physics for poets' variety, but I can imagine recommending it to a suitably warned nonscientist colleague as a fine way to get a quick and literate glimpse of the things that preoccupy physicists these days.

I say suitably warned because the clarity of the many sketches is uneven. In view of the vast scope this is hardly surprising. When subtle and intricate matters are characterized in a page or two there is a high risk that essential points may end up in the wrong place or be omitted altogether. This can be corrected in the give and take of a discussion group, but it can blemish a printed text. Sometimes omissions are unavoidable, but the author of such a book should hold fast to one rule: readers should always know when they are being told to take something on faith and when they are expected to figure it out for themselves. In the latter case the author has a moral obligation to give them the wherewithal to do it. Newton is not always true to this principle.

In the handful of pages on special relativity, for example, he tries to deduce the length contraction from what is known 'in the trade' as the invariance of the interval - a starting point that is algebraically simple to state, but entirely unintuitive. Although I believe he wants the reader to accept this as a postulate, he says nothing to forestall a conscientious reader from worrying why it is not evident. $\mathrm{He}$ then compounds the confusion by extracting length contraction from the invariance of the interval by an argument that can make sense only to a reader who understands the relativity of simultaneity, which he has yet to mention. When he does come to it (on the next page) he remarks that readers may find it shocking. But they had to understand it implicitly to make any sense of the earlier argument.

This is an example of a pedagogical lapse that could have been repaired. In other cases I suspect the concepts are so subtle and the treatment so sketchy that the sense of puzzlement the reader will surely acquire cannot be relieved by a simple reorganization of the argument.

So in recommending the book to a friend I would urge her to think hard, line-by-line, about what she was reading, but I would warn her that when some hard thought failed to clarify an obscure point, she should not assume that the fault was hers.

"Newton's book is, quite simply, a masterpiece," exudes Sheldon Glashow on the dust jacket in a noteworthy example of the hype that (almost) launched ten thousand $\mathrm{GeV}$, "I wish that I had written it". A masterpiece it isn't - not even a tour de force. But I respect Newton's accomplishment. Those few parts of it I could have written I like to think I would have done better, but most of it I couldn't have written at all. This is a noble but flawed attempt to do what nobody else has come close to doing. I hope it will inspire somebody to do even better.

David Mermin is at the Laboratory of Atomic and Solid State Physics, Cornell University. 532A Clark Hall, Ithaca, New York 14853 , USA.
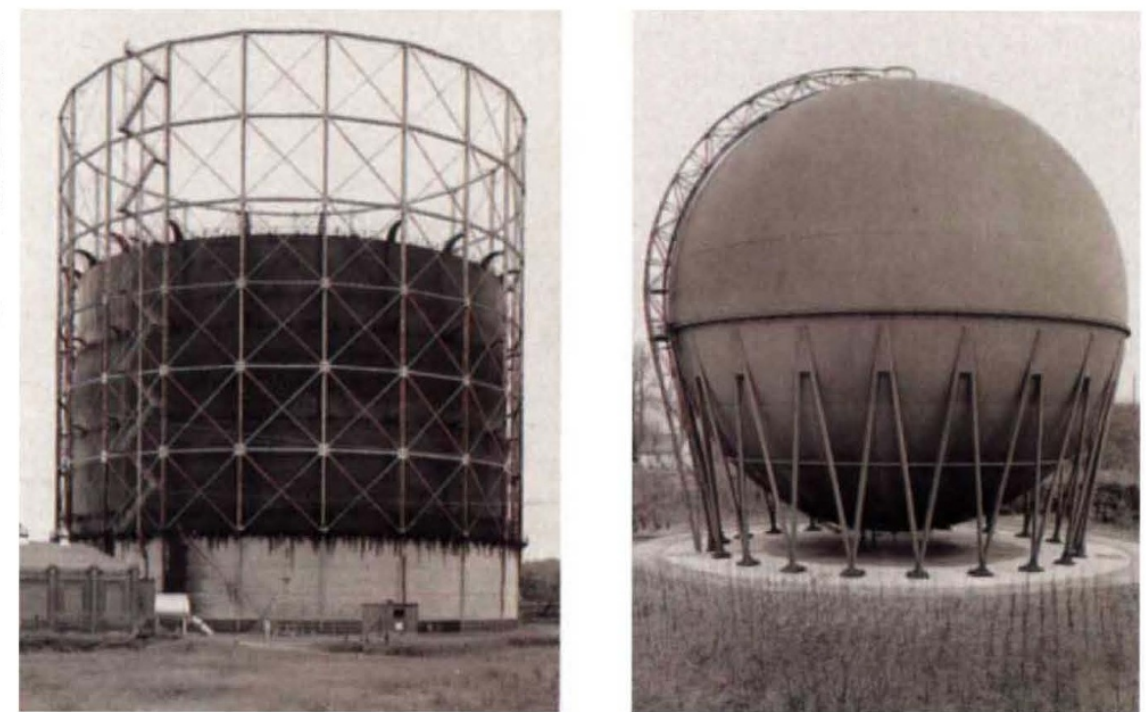

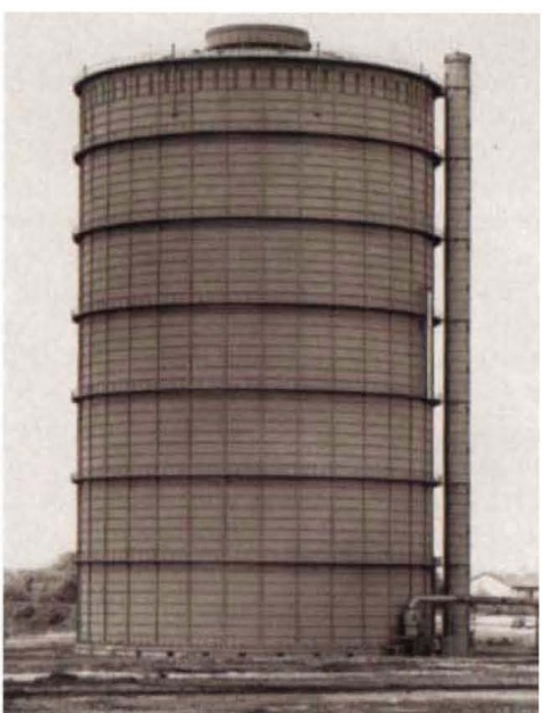

liquefied gas under pressure. The piston type (right) is a large, fixed cylinder in which a piston disk floats on the gas. From Gas Tanks by B. and H. Becher. MIT Press, £49.50, $\$ 55$.
WHAT a gas - three types of gas tanks (or gasometers). The oldest is the multiple section type (left). It works on the same principle as a bell jar upside down in a tank of water. Spherical gas tanks store 